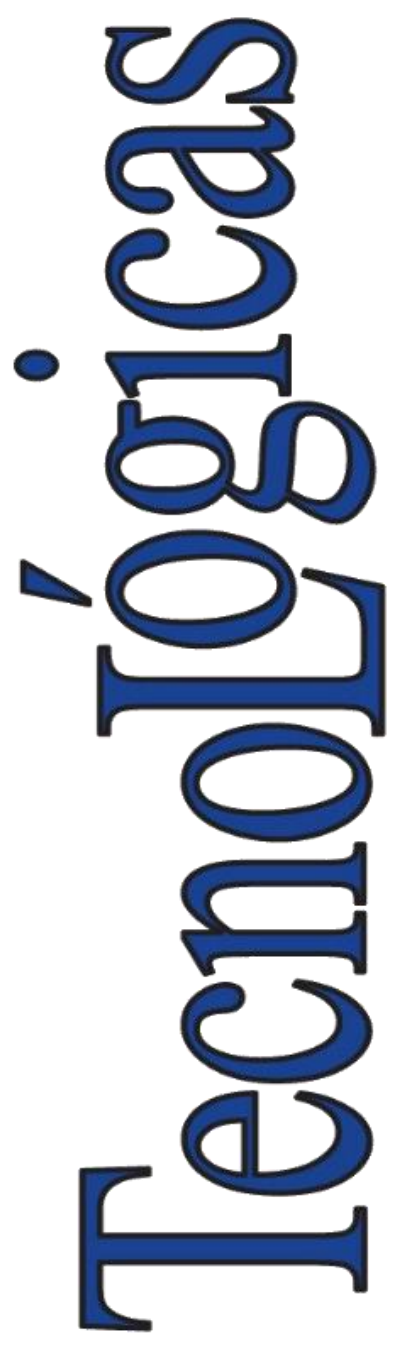

ISSN-p: 0123-7799 ISSN-e: $2256-5337$

Vol. 24, nro. 51, e1740, 2021

Recibido: 25 agosto 2020 Aceptado: 22 enero 2021 Disponible: 26 abril 2021

CInstituto Tecnológico Metropolitano Este trabajo está licenciado bajo una Licencia Internacional Creative Commons Atribución (CC BY-NC-SA)

\section{Revalorización de residuos de equipos eléctricos y electrónicos en Colombia: una alternativa para la obtención de metales preciosos y metales para la industria}

\section{Waste Electrical and Electronic Appliances Valorization in Colombia: An Alternative for Obtaining Precious Metals and Metals Used in Industry}

\author{
Carlos E. Aristizábal-Alzate1; \\ (D) José L. González Manosalva²; \\ (D) Andrés F. Vargas ${ }^{3}$;
}

\begin{abstract}
1Instituto Tecnológico Metropolitano, Medellín -Colombia, carlosaristizabal207157@correo.itm.edu.co 2Instituto Tecnológico Metropolitano, Medellín -Colombia, josegonzalez@itm.edu.co

${ }^{3}$ Instituto Tecnológico Metropolitano, Medellín -Colombia, andresvargas@itm.edu.co
\end{abstract}

Cómo citar / How to cite

C. E. Aristizábal-Alzate; J. L. González-Manosalva; A. F. Vargas, "Revalorización de residuos de equipos eléctricos y electrónicos en Colombia. Una alternativa para la obtención de metales preciosos y metales para la industria”, TecnoLógicas, vol. 24, nro. 51, e1740, 2021. https://doi.org/10.22430/22565337.1740

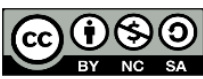




\section{Resumen}

El objetivo de este artículo es revisar el contexto mundial, y especialmente el colombiano, respecto al manejo de los residuos de equipos eléctricos y electrónicos, su aprovechamiento, recuperación y los principales métodos para la extracción de metales base y preciosos de alto valor agregado. Para ello, se realizó una revisión bibliográfica para obtener las cantidades de residuos, sus componentes principales y los métodos de extracción de metales base y preciosos; para Colombia, se realizó un estudio de caso, en el que, mediante un proceso de hidrometalurgia aplicado a teléfonos celulares, se calcularon las cantidades de metales base y preciosos que se pueden obtener. Se encontró que solo se aprovecha el 15,5\% de los residuos electrónicos en el mundo, que los principales componentes reciclables son el hierro (Fe), el cobre $(\mathrm{Cu})$, el aluminio $(\mathrm{Al})$, el plomo $(\mathrm{Pb})$, el níquel $(\mathrm{Ni})$, la plata $(\mathrm{Ag})$, el oro $(\mathrm{Au})$ y el paladio $(\mathrm{Pd})$, y que el uso de los residuos electrónicos como fuente de metales podría reducir el consumo de energía entre 60 \% y 95 \%. Para el estudio de caso en Colombia, se encontró que se podrían obtener 3,8 t/año de $\mathrm{Cu}, 3,5$ t/año de $\mathrm{Fe}, 56,5 \mathrm{~kg} / \mathrm{año}$ de $\mathrm{Ag}, 6 \mathrm{~kg} / \mathrm{año} \mathrm{de} \mathrm{Pd}$ y 10 $\mathrm{kg} / \mathrm{año} \mathrm{de} \mathrm{Au.} \mathrm{Se} \mathrm{concluye} \mathrm{que} \mathrm{es} \mathrm{viable} \mathrm{la} \mathrm{recuperación} \mathrm{de} \mathrm{metales} \mathrm{a} \mathrm{partir} \mathrm{de} \mathrm{residuos}$ eléctricos y electrónicos y que se deben buscar alternativas para aprovecharlos debido a su potencial valor agregado.

\section{Palabras clave}

Proceso químico, hidrometalurgia, recuperación de residuos, desarrollo sostenible.

\section{Abstract}

This article aims to review the global context, and especially the Colombian context, regarding the management of waste electrical and electronic appliances, their use, recovery and the main extraction methods for high added-value base and precious metals. A literature review was carried out to obtain the quantities of residues, their main components and the extraction methods for base and precious metals; for Colombia, a case study was carried out in which, through a hydrometallurgy process applied to cellular phones, the quantities of base and precious metals that can be obtained were calculated. Only $15.5 \%$ of e-waste in the world is recycled, the main recyclable components are iron $(\mathrm{Fe})$, copper $(\mathrm{Cu})$, aluminum $(\mathrm{Al})$, lead $(\mathrm{Pb})$, nickel $(\mathrm{Ni})$, silver $(\mathrm{Ag})$, gold $(\mathrm{Au})$ and palladium $(\mathrm{Pd})$, and that using e-waste as a source of metals could reduce energy consumption by 60-95\%. For the case study in Colombia, it was found that $3.8 \mathrm{t}$ /year of $\mathrm{Cu}, 3.5 \mathrm{t} /$ year of $\mathrm{Fe}, 56.5 \mathrm{~kg} /$ year of $\mathrm{Ag}, 6 \mathrm{~kg} /$ year of $\mathrm{Pd}$ and $10 \mathrm{~kg} / \mathrm{year}$ of Au could be obtained. The conclusion is that recovering metals from electrical and electronic waste is feasible and that alternatives should be sought to take advantage of them due to their potential added value.

\section{Keywords}

Chemical process, hydrometallurgy, waste recovery, sustainable development. 


\section{INTRODUCCIÓN}

Los residuos de equipos eléctricos y electrónicos (WEEE) son un problema mundial, ya que constituyen, en los países ricos, el $8 \%$ del total de los residuos sólidos municipales y, como promedio mundial, representan entre el $1 \%$ y el $3 \%$ [1], [2], [3]. Además, los componentes de estos son los que más crecimiento tienen [2], [4] con una tasa del $3 \%$ al $5 \%$ por año, y una producción anual, en promedio, de entre 20 y 50 millones de toneladas (Mt) [2], [5].

En la Tabla 1 se ilustran los aportes para algunos países desarrollados en el tema de WEEE y cómo se espera que sea el progreso y la evolución en la generación de este tipo de residuos hasta el 2020.

Tabla 1. Crecimiento global de los WEEE. Fuente: [3].

\begin{tabular}{lccccc}
\hline País & $\begin{array}{c}\text { Cantidad de } \\
\text { dispositivos en el } \\
\text { Mercado para el } \\
2012(\mathrm{Mt})\end{array}$ & $\begin{array}{c}\text { WEEE } \\
\text { estimado para } \\
\text { el } 2013(\mathrm{Mt})\end{array}$ & $\begin{array}{c}\text { WEEE por } \\
\text { persona } \\
\text { (kg/persona) }\end{array}$ & $\begin{array}{c}\text { WEEE estimado } \\
\text { para el 2020 } \\
(\mathrm{Mt})\end{array}$ & $\begin{array}{c}\text { Incremento } \\
(2013-2020)\end{array}$ \\
\hline EU-28 & 9800 & 10205 & 19,6 & 11430 & $12 \%$ \\
USA & 9350 & 9359 & 29,3 & 10050 & $7 \%$ \\
China & 12405 & 6033 & 4,4 & 12066 & $98 \%$ \\
Japón & 3300 & 3022 & 23,8 & 3200 & $5 \%$ \\
India & 3026 & 2751 & 2,2 & 6755 & $145 \%$ \\
Alemania & 1752 & 1696 & 21,9 & 1974 & $16 \%$ \\
Rusia & 1599 & 1556 & 10,9 & 2000 & $28 \%$ \\
Brasil & 1850 & 1530 & 7,1 & 1850 & $20 \%$ \\
Francia & 1520 & 1224 & 21,6 & 1625 & $32 \%$ \\
Italia & 1124 & 1154 & 19,3 & 1343 & $16 \%$ \\
Corea & 959 & 961 & 19,2 & 1050 & $9 \%$ \\
Turquía & 726 & 661 & 8,8 & 800 & $21 \%$ \\
Holanda & 432 & 394 & 23,3 & 421 & $6 \%$ \\
Rumania & 217 & 157 & 7,9 & 227 & $44 \%$ \\
Noruega & 175 & 127 & 25,8 & 136 & $7 \%$ \\
Bulgaria & 86 & 62 & 8,6 & 89 & $43 \%$ \\
\hline
\end{tabular}

La problemática radica en que los WEEE no son dispuestos de una manera adecuada; por ejemplo, de los 41,8 Mt de WEEE generados en todo el mundo en el año 2014, el sistema de recolección de residuos sólidos tan solo colectó y dispuso correctamente 6,5 Mt de desechos electrónicos, mientras que el resto de los desechos electrónicos fueron arrojados a rellenos sanitarios, incinerados, desechados sin ningún control [2], [6], [7] o fueron almacenados por sus propietarios por largos periodos de tiempo [6]. Por ejemplo, a nivel mundial, entre el 2004 y 2014, fueron vendidos 14 mil millones de unidades de celulares, de los cuales menos del $3 \%$ fueron recuperados; en países desarrollados como Alemania, solamente el $5 \%$, y en China, menos del $1 \%$ fue recuperado [8], [9].

Por lo tanto, esto se convierte en un problema ambiental de índole mundial, debido a que los dispositivos eléctricos y electrónicos (televisores, circuitos impresos, celulares, máquinas de copiado, monitores LED/LCD, etc.) tienen una vida útil más corta [3], [6], [10], [11] porque hoy en día la tecnología avanza rápidamente y los consumidores de este tipo de productos necesitan y demandan dispositivos más avanzados y con una mayor rapidez [12], [13], [14]. 
A lo anterior hay que sumarle el hecho de que los países con mayor ingreso per cápita y con economías desarrolladas están acelerando la generación de este tipo de residuos, dado que las personas poseen mejores ingresos, lo que les permite cambiar constantemente de equipos electrónicos [3], [15]. En cifras globales, en el 2014 se generaron por persona 5,9 kg de WEEE y $6,1 \mathrm{~kg}$ en el 2016, lo que quiere decir que este indicador seguirá aumentando su valor [7], [16].

\section{MINERÍA URBANA: RECICLAJE Y APROVECHAMIENTO DE LOS WEEE}

El reciclaje de residuos de fuentes urbanas se está posicionando como una opción sostenible y rentable de obtención de materias primas de alto valor agregado [9], [12], [17] porque en muchos casos la concentración de estos recursos es mucho mayor en el residuo que en la fuente natural [1], [6]. Por ejemplo, los niveles de metales preciosos en WEEE son mayores en aproximadamente 40 veces, si se compara con la minería convencional; un depósito primario tiene alrededor de cinco gramos de oro por tonelada, mientras que los dispositivos electrónicos contienen aproximadamente $280,0 \mathrm{~g}$ y las tarjetas electrónicas impresas individuales (PCB), hasta 1400,0 g de oro por tonelada de residuos [12], [14], [18] (ver Tabla 2). Las proporciones aquí descritas son semejantes para los casos del paladio, el platino y el galio [14].

Además, se tienen grandes beneficios ambientales, ya que se evitaría el uso de sustancias químicas tóxicas convencionalmente usadas en la minería tradicional; por ejemplo, el agua regia, la cual es altamente corrosiva [19], [20].

Tabla 2. Varios tipos de WEEE y sus contenidos de metales. Fuente: modificado de [12].

\begin{tabular}{|c|c|c|c|c|c|c|c|c|}
\hline E-Waste & $\begin{array}{c}\text { Fe (wt } \\
\%)\end{array}$ & $\begin{array}{c}\mathrm{Cu}(\mathrm{wt} \\
\%)\end{array}$ & $\begin{array}{c}\mathrm{Al}(\mathrm{wt} \\
\%)\end{array}$ & $\begin{array}{c}\mathrm{Pb}(\mathrm{wt} \\
\%)\end{array}$ & $\begin{array}{c}\mathrm{Ni}(\mathrm{wt} \\
\%)\end{array}$ & $\begin{array}{c}\mathrm{Ag} \\
(\mathrm{ppm})\end{array}$ & $\begin{array}{c}\mathrm{Au} \\
(\mathrm{ppm})\end{array}$ & $\begin{array}{c}\mathrm{Pd} \\
(\mathrm{ppm})\end{array}$ \\
\hline Tableros de TV & 28 & 10 & 10 & 1,0 & 0,3 & 280 & 20 & 10 \\
\hline Tableros PC & 7 & 20 & 5 & 1,5 & 1 & 1000 & 250 & 110 \\
\hline Desechos de teléfonos móviles & 5 & 13 & 1 & 0,3 & 0,1 & 1380 & 350 & 210 \\
\hline Dispositivos de audio & 23 & 21 & 1 & 0,14 & 0,03 & 150 & 10 & 4 \\
\hline Reproductores de DVD & 62 & 5 & 2 & 0,3 & 0,05 & 115 & 15 & 4 \\
\hline Desechos de calculadora & 4 & 3 & 5 & 0,1 & 0,5 & 260 & 50 & 5 \\
\hline Tableros principales de PC & 4,5 & 14,3 & 2,8 & 2,2 & 1,1 & 639 & 566 & 124 \\
\hline $\begin{array}{l}\text { Desechos de circuitos impresos de } \\
\text { tableros }\end{array}$ & 12 & 10 & 7 & 1,2 & 0,85 & 280 & 110 & - \\
\hline Desechos de TV & - & 3,4 & 1,2 & 0,2 & 0,038 & 20 & $<10$ & $<10$ \\
\hline Desechos electrónicos & 8,3 & 8,5 & 0,71 & 3,15 & 2,0 & 29 & 12 & - \\
\hline Desechos de PC & 20 & 7 & 14 & 6 & 0,85 & 189 & 16 & 3 \\
\hline Desecho electrónico típico & 8 & 20 & 2 & 2 & 2 & 2000 & 1000 & 50 \\
\hline Desechos electrónicos: Muestra 1 & 37,4 & 18,2 & 19 & 1,6 & - & 6 & 12 & - \\
\hline Desechos electrónicos: Muestra 2 & 27,3 & 16,4 & 11,0 & 1,4 & - & 210 & 150 & 20 \\
\hline Circuitos impresos de tableros & 5,3 & 26,8 & 1,9 & - & 0,47 & 3300 & 80 & - \\
\hline Desechos electrónicos (1972) & 26,2 & 18,6 & - & - & - & 1800 & 220 & 30 \\
\hline Mezcla WEEE & 36 & 4,1 & 4,9 & 0,29 & 1,0 & - & - & - \\
\hline
\end{tabular}

Nota: “-”, No se encuentra reportado. 
La mayor parte de los elementos químicos enumerados en la Tabla 2 se utilizan para aplicaciones de alta tecnología o son instalados en los equipos eléctricos y electrónicos. La principal razón para el peligro de la disponibilidad y la demanda de estos materiales es la falta o carente implementación de estrategias de reciclaje de WEEE [9], [13], [21].

Adicionalmente, la recuperación de recursos valiosos implica contar con tecnologías eficientes para la ejecución de las operaciones unitarias en las etapas iniciales de reciclaje de los WEEE, ya que cuentan con una matriz plástica de bajo valor [22], por lo que cerca del $80 \%$ del valor económico se encuentra concentrado en los metales preciosos y estos tan solo son el $1 \%$ del total [3], [23]. Se necesitan procesos de recuperación que no emitan sustancias tóxicas a la atmósfera, como los bromuros de llama retardante, y evitar que se contaminen las fuentes de agua en los procesos subsecuentes de recuperación y extracción de los materiales metálicos de interés [21], [22], [24]. Por esto es que se deben incentivar propuestas encaminadas a [25]: el diseño y la implementación de las estrategias dirigidas a los usuarios o consumidores de aparatos eléctricos y electrónicos para lograr la eficiencia en la devolución y recolección de los WEEE; y promover la incorporación y reintegración de los componentes, partes o materiales obtenidos de los WEEE en los ciclos económicos y productivos del país.

Por otro lado, los beneficios ambientales son más que considerables, ya que el reciclaje de WEEE para la recuperación de metales preciosos y metales demandados por la industria en general, como el cobre $(\mathrm{Cu})$ y el aluminio $(\mathrm{Al})$, ayuda a mantener las fuentes primarias intactas, eliminando todos los daños colaterales que trae la minería convencional, disminuyendo la cantidad de desperdicios generados por una población al darle valor y uso a los WEEE, eliminando la polución causada por los metales pesados contenidos en estos [12], [13], [14], [19], [26], [27], [28], [29], evitando así la afectación a la salud pública de metales neurotóxicos que se encuentran en grandes cantidades en residuos como el plomo (Pb) [30], [31].

Adicionalmente, el consumo energético es menor que en los procesos de extracción convencionales (ver Tabla 3) [12], [13], [14], [21], [28], lo que se vería reflejado en menores emisiones de gases de efecto invernadero a la atmosfera y de contaminantes [20], [27], [32].

Tabla 3. Ahorro de energía mediante el reciclaje de los metales/materiales en comparación con la extracción convencional de los recursos primarios. Fuente: [12].

\begin{tabular}{cc}
\hline Material & Ahorro de energía (\%) \\
\hline Aluminio & 95 \\
Cobre & 85 \\
Hierro y acero & 74 \\
Plomo & 65 \\
Zinc & 60 \\
Papel & 64 \\
Plásticos & $>80$ \\
\hline
\end{tabular}

Adicionalmente, se cuenta con que los metales base y preciosos recuperados son tranzados como materias primas de alta importancia en los mercados internacionales [16], lo que sustentaría la viabilidad económica de este tipo de aprovechamiento de residuos. En la Figura 1 se ilustran los precios (USD/kg) de mercado para los metales base y los preciosos. 


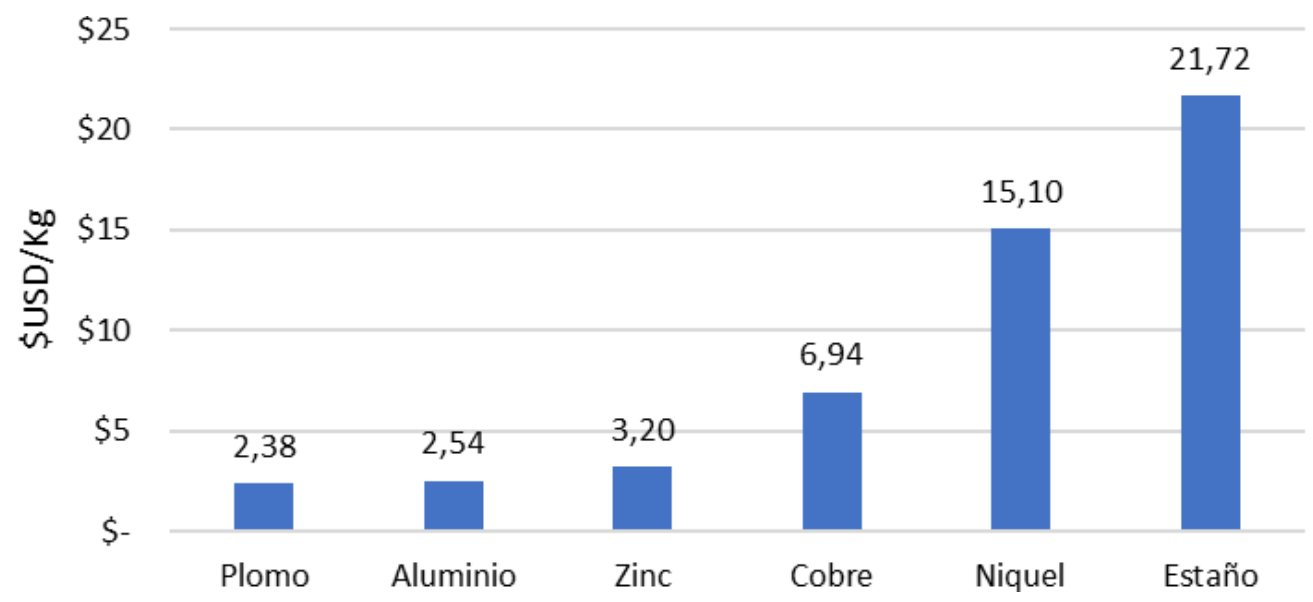

(a)

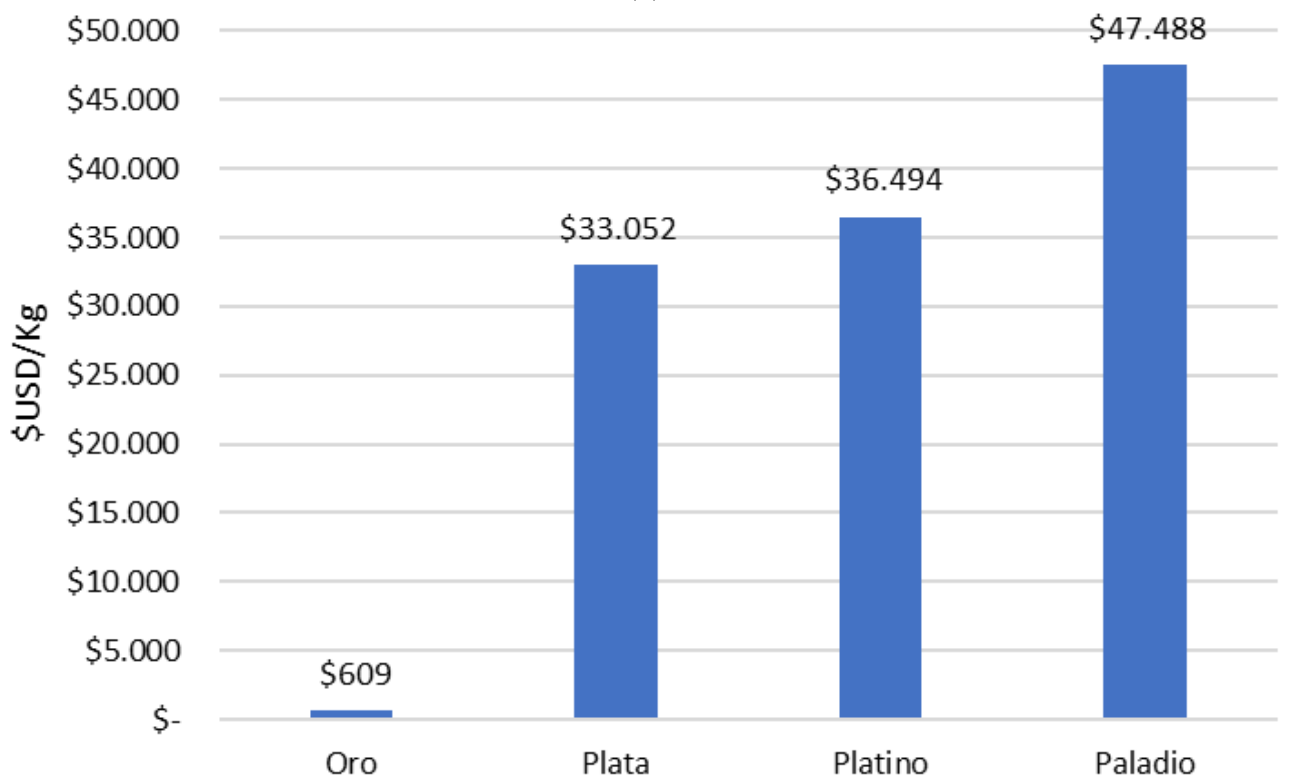

(b)

Figura 1. Precio ( $\$ \mathrm{USD} / \mathrm{kg}$ ) de los metales base (a) y preciosos (b). Fuente: modificado de [33].

Aunque el plástico sea cercano al $30 \%$ o $35 \%$ del peso del WEEE, la generación de valor de este componente es mínima debido a la heterogeneidad de los materiales que lo componen, lo que dificulta su separación y reciclaje [34]-[36].

Adicionalmente, es difícil su reutilización debido a que este tipo de plásticos contienen sustancias muy contaminantes, tales como bromuros de llama retardante o los contaminantes orgánicos persistentes [18], [37], [38].

\section{SITUACIÓN DEL RECICLAJE DE RESIDUOS ELECTRÓNICOS EN COLOMBIA}

Colombia es un gran productor en la región de desechos electrónicos y se considera que el problema ambiental será cada vez mayor debido a la mala disposición de los WEEE y a las sustancias tóxicas contenidos en estos, tales como los bromuros de llama retardante, los metales pesados, las resinas epóxicas y los plásticos [13], [14], [26]. Por estas razones, desde el año 2010 en el país se trabaja en diseñar, implementar y consolidar las directrices 
relacionadas con la disposición final adecuada de los WEEE, basados en la responsabilidad extendida del productor, la cual consiste en que fabricantes e importadores deben hacerse cargo de los productos que pusieron en el mercado una vez son desechados o culmina su vida útil. Por tal motivo, en el año 2013 es sancionada la Ley 1672 sobre gestión integral de residuos de aparatos eléctricos y electrónicos. Sin embargo, para el caso de tablets y computadores desechados en el año 2013, solo se recolecto el $25 \%$ de los residuos generados por estos productos y, para ese mismo año, se acumuló entre 80000 y 140000 toneladas de WEEE en el país [28]. Para el caso de dispositivos móviles, desde el año 2007, a través de la iniciativa público-privada Recicla tu móvil o celular y comunícate con la Tierra, establecida entre el gobierno, operadores de servicios móviles y otros sectores de la industria relacionados, se contribuyó a gestionar 627750 celulares, 378855 baterías y 1993969 accesorios en el transcurso de dos años. Una vez finalizada la campaña, los operadores continuaron desarrollando la iniciativa, logrando gestionar, entre 2007 y 2014, alrededor 907613 teléfonos móviles, 2931759 accesorios y 703463 baterías, lo que equivale a 185 toneladas de WEEE [39].

Los WEEE reciclados en Colombia son recolectados en puntos de acopio y entregados a los fabricantes e importadores o gestores especializados [40], y al tener una industria tan incipiente en el campo de la electrónica y tener que importar la mayoría de dispositivos [28], casi todos estos son devueltos a los países de origen de los fabricantes, como por ejemplo a alguno de los 35 miembros de la Organización para la Cooperación y el Desarrollo Económicos (OCDE) [24], llevándose el potencial valor agregado que representan los metales contenidos en estos y una posibilidad de generar una industria sustentable, rentable y amigable con el medio ambiente [28], [41]. Por su parte, en Colombia, de 45000 toneladas de computadores que habían acumuladas a 2007, se podrían extraer 3000 toneladas de cobre, lo que equivale a más de 25 millones de USD (según precios de cobre del inicio de 2008) [28], esto sin contar los metales preciosos contenidos en estos residuos electrónicos [42]. Sin embargo, la recuperación de metales en el país es incipiente, ya que la industria es inmadura y en muchos casos es desarrollada de manera informal [43].

Por estas razones es que el Gobierno Nacional ha establecido políticas públicas que fomenten la recolección de WEEE, buscando garantizar el derecho colectivo de gozar de un ambiente sano y el deber de proteger la diversidad e integridad del ambiente, planificar el manejo y aprovechamiento de los recursos naturales a fin de garantizar su desarrollo sostenible. Para esto, se expidió el Decreto 284 de 2018, en el cual se establecen las directrices, para realizar un adecuada recolección de los WEE, su transporte y gestión final del residuo, la cual puede ser una disposición final integral para el aprovechamiento y reciclaje de piezas funcionales o para la obtención de materiales y, de este modo, reincorporarlos en los ciclos económicos y productivos del país [25], [44]. Sin embargo, esto solamente se logra con una vinculación, un conocimiento claro de los alcances, obligaciones, responsabilidades de cada uno de los actores involucrados en la cadena y el ciclo de vida de los aparatos eléctricos y electrónicos [20], [45].

Por lo tanto, para que este tipo de medidas en pro de la recuperación reincorporación de metales se presenten de forma efectiva en el ciclo productivo, se debe establecer una armonía y compromiso de diferentes actores a lo largo de la cadena de valor y ciclo de vida de estos equipos electrónicos, ya que en países subdesarrollados las técnicas de recuperación siguen siendo artesanales y realizadas con bajos estándares de calidad y de tecnificación debido a la falta de legislación y políticas públicas acertadas [7], [46]. En la Figura 2 se presenta el flujo de decisiones y procesos dentro de la gestión integral de los WEE en Colombia, y muestra las diferentes etapas y límites del sistema que tienen los diferentes actores involucrados en la correcta disposición de estos residuos. 


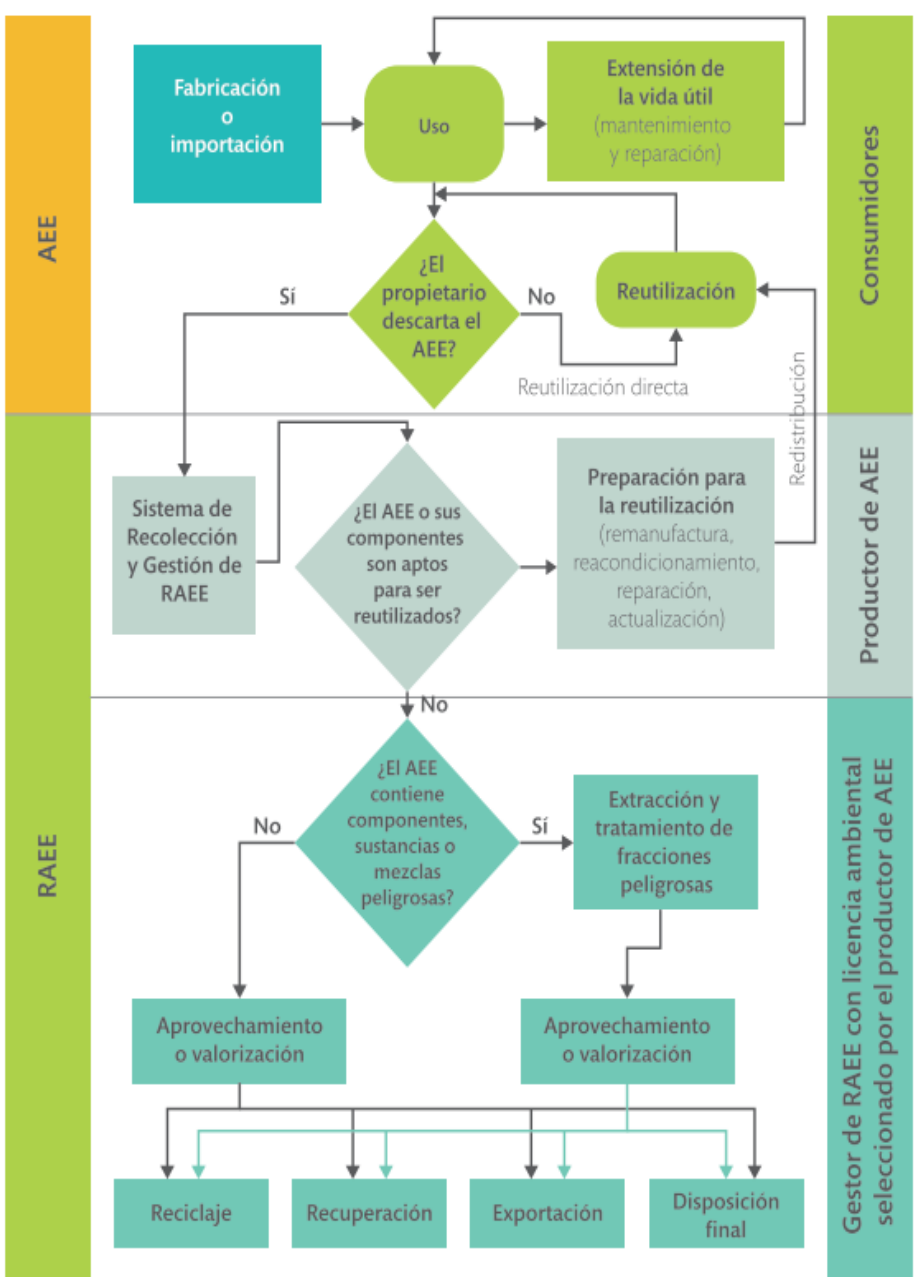

Figura 2. Esquema del sistema gestión integral de los WEEE en Colombia. Fuente: [40].

A pesar de que, se describe un esquema de la cuna a la tumba, es algo lineal y de poca interactividad entre actores, incluso dejan por fuera a otros actores; por ejemplo, del sector industrial, los cuales pueden presentar nuevas ideas sobre reaprovechamiento y generación de valor a partir de los WEEE. Por lo tanto, es importante considerar dentro del esquema la aparición de nuevos actores que propongan nuevas rutas de disposición final o revalorización. Por ejemplo, la recuperación de metales base y preciosos a partir de los WEEE, cuyas metodologías son descritas a continuación.

\section{METOdOlogías PARA la RECUPERACIÓN DE METALES PRECIOSOS Y BASE}

La tecnología de procesamiento que se aplica para la recuperación de metales base y preciosos a partir de desechos electrónicos está impulsada por su impacto ambiental o las regulaciones locales. Sin embargo, debe de considerar aspectos relacionados con la importancia o aplicabilidad económica-industrial, como lo es el costo de procesamiento, los posibles valores de salida de los desechos y las ganancias ocasionadas por la venta de los metales recuperados [31], [47]. A saber, hay que tener en cuenta que las ganancias del proceso hidrometalúrgico son mayores (USD $\$ 241,61 / \mathrm{kg}$ ), seguidas por el electroquímico (USD $\$ 213,29 / \mathrm{kg}$ ) y, finalmente, por el pirometalúrgico (USD $\$ 179,91 / \mathrm{kg}$ ) [19]. 


\subsection{Descripción del proceso de recuperación de metales base y preciosos}

El proceso de recuperación inicia con un pretratamiento, el cual implica lavado, trituración, separación e incineración, dependiendo de la naturaleza de los desechos [38], [48]-[50]. Aunque los metales recuperados por lo general son de baja pureza, estos metales preciosos crudos son sometidos al proceso de refinación mejorando su pureza a más del 99,9\% mediante disoluciones repetidas, filtración, concentración y reducción [51].

Para la recuperación de metales preciosos a partir de residuos electrónicos, se proponen métodos convencionales (ver Figura 3), los cuales se basan en procesos físicos, biohidrometalúrgicos, hidrometalúrgicos y pirometalúrgicos [12], [29], [46], [52] y metodologías avanzadas como la electroquímica, la cual es considerada una tecnología prometedora, ya que permite recuperar metales con un mínimo uso de reactivos y productos químicos, altas eficiencias energéticas y muy bajas emisiones de contaminantes al medio ambiente [29].

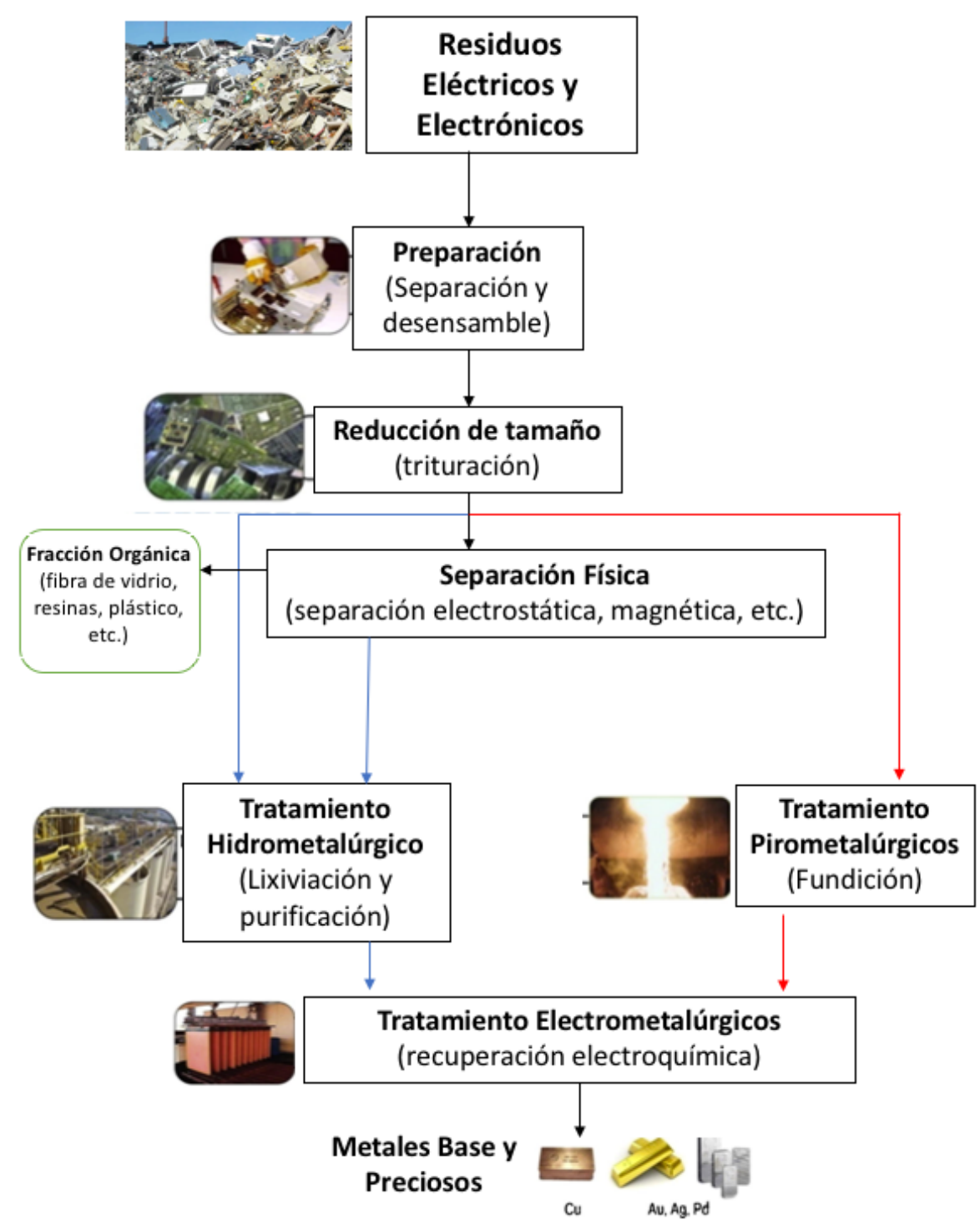

Figura 3. Diagrama con los posibles procesos y rutas para la recuperación de metales a partir de residuos electrónicos. Fuente: [12]. 
La mayoría de procesos de reciclaje y recuperación de metales a escala industrial son físicos y pirometalúrgicos; es decir, primero se deben separar varios componentes de los WEEE mediante clasificación, trituración, procesos magnéticos, etc., para concentrar los metales y aumentar el área superficial y luego proceder con el tratamiento térmico más complejo, el cual se basa en la diferencia de los puntos de fusión de los diferentes metales para lograr una separación secuencial mientras se presenta el aumento de la temperatura [6], [12], [51]. A pesar de que este tipo de tratamiento térmico de los WEEE se encuentra ampliamente difundido, requiere de altos consumos de energía [24], grandes inversiones de capital y debe realizarse en instalaciones que procesen grandes cantidades de WEEE para que el proceso sea financieramente sostenible [6], [19], pues, de no hacerse correctamente, puede exponer a las personas a sustancias tóxicas [30], [53].

En la actualidad se prefiere un tratamiento físico seguido de uno hidrometalúrgico, ya que es un proceso más amigable con el medio ambiente que el pirometalúrgico, porque i) durante el proceso no se emiten a la atmosfera químicos tóxicos, como dioxinas, furanos, metales volátiles, $\mathrm{Cl}_{2}, \mathrm{Br}_{2}, \mathrm{SO}_{2}$ y $\mathrm{CO}_{2}$; ii) se tiene un bajo consumo de energía; (iii) se alcanzan altos rendimientos en la recuperación de metales; (iv) no hay generación de escoria, excepto algunos plásticos; y (v) se cuenta con condiciones favorables y fáciles de trabajo y operación [29], [34], [54].

El proceso hidrometalúrgico comienza con una lixiviación de los WEEE con ácido sulfúrico, para posteriormente seguir con una separación sólido-líquido. El líquido o filtrado obtenido en la etapa anterior es una solución que se encuentra enriquecida en cobre, el cual es recuperado mediante tratamientos químicos (cementación, coagulación, precipitación, etc.) [38].

Por otro lado, el sólido residual retenido en la separación sólido-líquido, es llevado a un proceso de lixiviación con tiourea o cianuro para la recuperación de metales preciosos, tales como el oro y la plata [35], [55], [56]. También para este propósito son utilizados haluros y otros ácidos fuertes $\left(\mathrm{HCl}\right.$ y $\left.\mathrm{HNO}_{3}\right)$ [46], [57], [58]. Sin embargo, estos reactivos químicos son considerados tóxicos y nocivos para la salud humana y el ambiente, por lo que se han buscado nuevas posibilidades o nuevos químicos que reemplacen estas sustancias. Hasta ahora, se ha estudiado el cloruro cúprico $\left(\mathrm{CuCl}_{2}\right)$ y el persulfato de potasio $\left(\mathrm{K}_{2} \mathrm{~S}_{2} \mathrm{O}_{8}\right)$ [56] el tiosulfato $\left(\left(\mathrm{NH}_{4}\right)_{2} \mathrm{~S}_{2} \mathrm{O}_{3}\right)$ y el persulfato de amonio $\left(\left(\mathrm{NH}_{4}\right)_{2} \mathrm{~S}_{2} \mathrm{O}_{8}\right)$ [5], [56], [59].

A pesar de todos las discusiones y preocupaciones por el ambiente que ha suscitado la aplicación de la hidrometalurgia para la recuperación de metales preciosos y de interés industrial, se puede afirmar que este procedimiento es el más rápido y eficiente que hay a escala industrial, y es más limpio y amigable con el ambiente si se compara con otras metodologías usadas para este fin [12], [17], [60]. En la Figura 4 se ilustra la metodología que se aplica para el caso de estudio descrito en el numeral 4, el cual consiste en la recuperación de metales base y preciosos de residuos de teléfonos móviles en Colombia. 


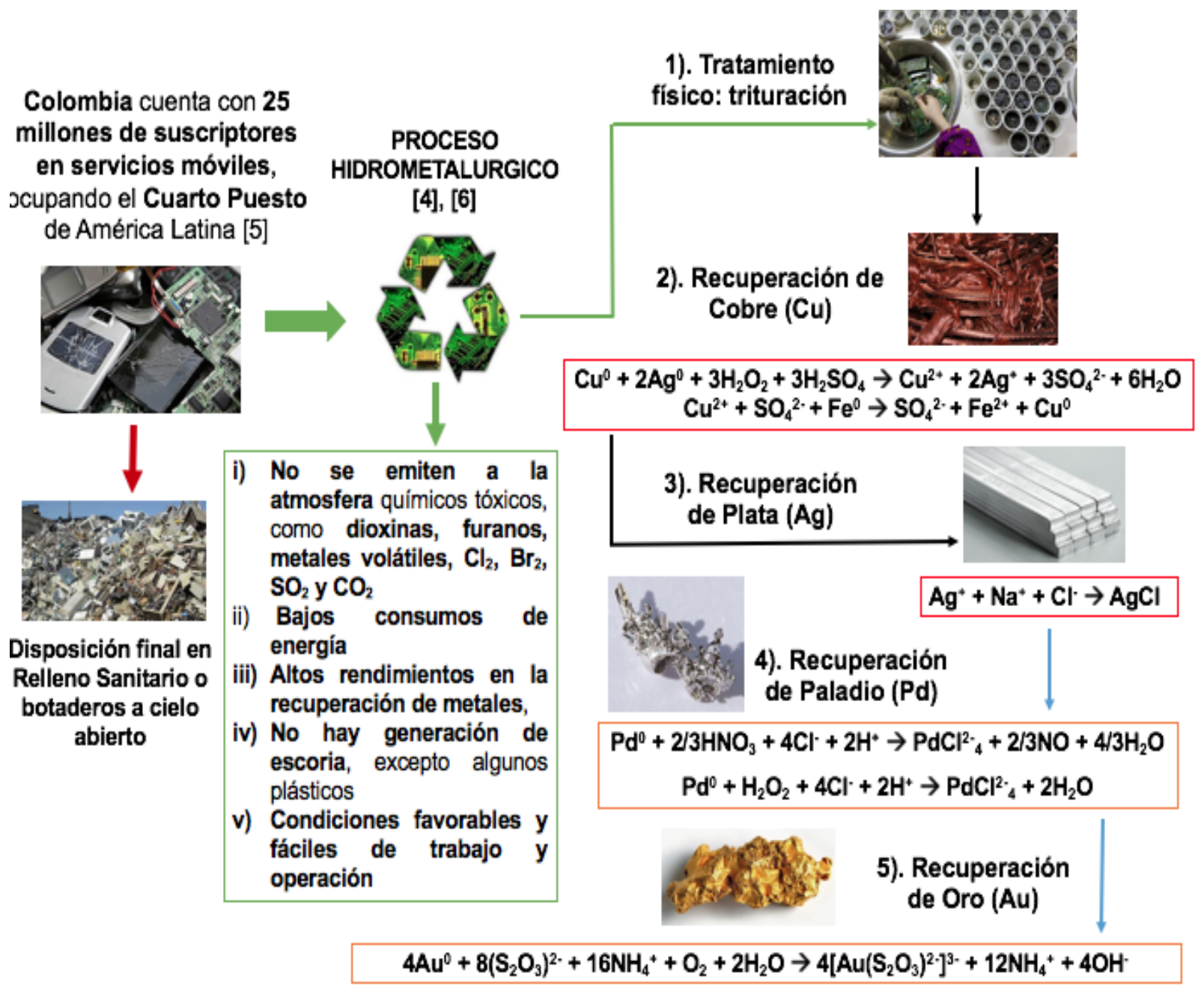

Figura 4. Metodología para la recuperación de metales preciosos y de interés industrial a partir de WEEE y empleando un proceso hidrometalúrgico. Fuente: elaboración propia.

\subsection{Descripción del proceso hidrometalúrgico}

Inicialmente, los residuos eléctricos y electrónicos que contienen los metales de interés son fragmentados para reducir el tamaño de partícula, aumentando de esta forma el área superficial o el área de contacto sólido-líquido, lo que mejoraría el proceso hidrometalúrgico. Lo recomendado es tener un tamaño de partícula de 0,01 m [50], [60].

Primero, se deben recuperar los metales base que se encuentran en mayor cantidad, como, por ejemplo, el Cobre $(\mathrm{Cu})$, Aluminio (Al), Hierro ( $\mathrm{Fe})$, Níquel (Ni), entre otros. Para este propósito se propone una lixiviación con peróxido de hidrogeno $\left(\mathrm{H}_{2} \mathrm{O}_{2}\right)$ y ácido sulfúrico $\left(\mathrm{H}_{2} \mathrm{SO}_{4}\right)$, el cual tiene porcentajes de recuperación superiores al $95 \%$. Posterior a esto, se realiza una filtración, de la cual saldrá un líquido, en el que estará disuelto el Cu y un sólido en el que estarán presentes los metales preciosos $(\mathrm{Pd}, \mathrm{Ag}$ y $\mathrm{Au})$, y que irá al proceso de recuperación de Paladio ( $\mathrm{Pd})$. Sin embargo, de acuerdo con [5], la plata también es disuelta por estos dos reactivos, por lo que debe ser separada del $\mathrm{Cu}$ mediante una precipitación con $\mathrm{NaCl}$, seguido de una filtración. Las reacciones involucradas en el proceso de recuperación de $\mathrm{Cu}$ y $\mathrm{Ag}$ son las representadas en (1), (2) y (3). 


$$
\begin{gathered}
\mathrm{Cu}_{(\mathrm{s})}^{0}+2 \mathrm{Ag}_{(\mathrm{s})}^{0}+3 \mathrm{H}_{2} \mathrm{O}_{2}+3 \mathrm{H}_{2} \mathrm{SO}_{4} \rightarrow \mathrm{Cu}^{2+}{ }_{(\mathrm{aq})}+2 \mathrm{Ag}_{(\mathrm{aq})}+3 \mathrm{SO}_{4}^{2-}{ }_{(\mathrm{aq})}+6 \mathrm{H}_{2} \mathrm{O} \\
\mathrm{Ag}_{(\mathrm{aq})}+\mathrm{Na}_{(\mathrm{aq})}^{+}+\mathrm{Cl}_{(\mathrm{aq})} \rightarrow \mathrm{AgCl}_{(\mathrm{s})}+\mathrm{Na}^{+}{ }_{(\mathrm{aq})} \\
\mathrm{Cu}^{2+}{ }_{(\mathrm{aq})}+\mathrm{SO}_{4}^{2-}{ }_{(\mathrm{aq})}+\mathrm{Fe}_{(\mathrm{s})} \rightarrow \mathrm{SO}_{4}^{2-}{ }_{(\mathrm{aq})}+\mathrm{Fe}^{2+}{ }_{(\mathrm{aq})}+\mathrm{Cu}_{(\mathrm{s})}
\end{gathered}
$$

De acuerdo con [13] y [62], en el proceso de recuperación de Paladio ( $\mathrm{Pd}$ ) se debe utilizar una lixiviación con soluciones de $\mathrm{HCl} / \mathrm{NaCl}$ y $\mathrm{HNO}_{3} / \mathrm{H}_{2} \mathrm{O}_{2}$, permitiendo una recuperación de entre el $93 \%$ y el $95 \%$. A continuación, son ilustradas las reacciones químicas mediante la representación de (4) y (5).

$$
\begin{aligned}
& \mathrm{Pd}_{(\mathrm{s})}+2 / 3 \mathrm{HNO}_{3(\mathrm{l})}+4 \mathrm{Cl}_{(\mathrm{aq})}^{-}+2 \mathrm{H}^{+}{ }_{(\mathrm{aq})} \rightarrow \mathrm{PdCl}^{2-}{ }_{(\mathrm{aq})}+2 / 3 \mathrm{NO}_{(\mathrm{g})}+4 / 3 \mathrm{H}_{2} \mathrm{O}_{(\mathrm{l})} \\
& \mathrm{Pd}_{(\mathrm{s})}+\mathrm{H}_{2} \mathrm{O}_{2(\mathrm{aq})}+4 \mathrm{Cl}_{(\mathrm{aq})}+2 \mathrm{H}^{+}{ }_{(\mathrm{aq})} \rightarrow \mathrm{PdCl}^{2-}{ }_{(\mathrm{aq})}+2 \mathrm{H}_{2} \mathrm{O}_{(\mathrm{aq})}
\end{aligned}
$$

Por otro lado, para la recuperación del Oro (Au), [61] recomiendan una lixiviación con cianuro y tiourea, ya que son procesos que permiten obtener altos rendimientos en la recuperación de este metal precioso, son económicos y han sido ampliamente estudiados. Sin embargo, estos procesos generan gran cantidad de contaminantes, afectando drásticamente la salud y el medio ambiente [5], [56], [62].

Existen métodos que utilizan reactivos más inocuos, como son el tiosulfato ((NH4)2S2O3) o con persulfato de amonio ((NH4)2S2O8), ya que son de bajo costo, son amigables con el medio ambiente, promueven una tasa de lixiviación alta [5], [55], [59] y posibilitan una recuperación mayor al $93 \%$ del Au [63]. Este proceso químico es llevado a cabo en una solución de amonio-cobre (II)-tiosulfato, ya que debe ser catalizado por el cobre para que ocurra [59]. Las reacciones químicas involucrada son las ilustradas en (6) y (7):

$$
\begin{gathered}
4 \mathrm{Au}^{0}{ }_{(\mathrm{s})}+5\left(\mathrm{~S}_{2} \mathrm{O}_{3}\right)^{2-}{ }_{(\mathrm{aq})}+\mathrm{Cu}\left(\mathrm{NH}_{3}\right)_{4}{ }^{2+}{ }_{(\mathrm{aq})} \rightarrow 4\left[\mathrm{Au}\left(\mathrm{S}_{2} \mathrm{O}_{3}\right)_{2}\right]^{{ }^{--}{ }_{(\mathrm{aq})}}+\left[\mathrm{Cu}\left(\mathrm{S}_{2} \mathrm{O}_{3}\right)_{3}\right]^{5-}{ }_{(\mathrm{aq})}+4 \mathrm{NH}_{3} \\
8\left(\mathrm{~S}_{2} \mathrm{O}_{3}\right)^{2-}{ }_{(\mathrm{aq})}+2 \mathrm{Cu}\left(\mathrm{NH}_{3}\right)_{4}{ }^{2+}{ }_{(\mathrm{aq})} \rightarrow\left[2 \mathrm{Cu}\left(\mathrm{S}_{2} \mathrm{O}_{3}\right)_{3}\right]^{5-}{ }_{(\mathrm{aq})}+8 \mathrm{NH}_{3}
\end{gathered}
$$

La reacción química representada por (7), no solo reduce la concentración de ion cúprico, sino que también consume tiosulfato, los cuales son esenciales para la lixiviación del oro.

Como se desconoce la selectividad de cada una de las reacciones involucradas en el proceso de lixiviación de oro con tiosulfato y dado que este tipo de proceso requiere bastantes recursos, tiempo y equipos auxiliares, y ya que un inadecuado tratamiento de los subproductos podría ocasionar serios problemas para la salud y el ambiente [19], [51], se procede a evaluar un método emergente, el cual utiliza clorato de sodio $\left(\mathrm{NaClO}_{3}\right)$, como agente de cloración; ácido sulfúrico $\left(\mathrm{H}_{2} \mathrm{SO}_{4}\right)$, como agente de lixiviación; se emplea cloruro de sodio $(\mathrm{NaCl})$ para proporcionar el $\mathrm{Cl}^{-}$necesario para la formación de complejos con $\mathrm{Au}$; se usa metilisobutilcetona (MIBK) para extraer y transportar el oro de una fase acuosa a una orgánica; y ácido oxálico, para reducir y recuperar el oro. En la Figura 5 se muestra el mecanismo de extracción utilizando esta acetona. 


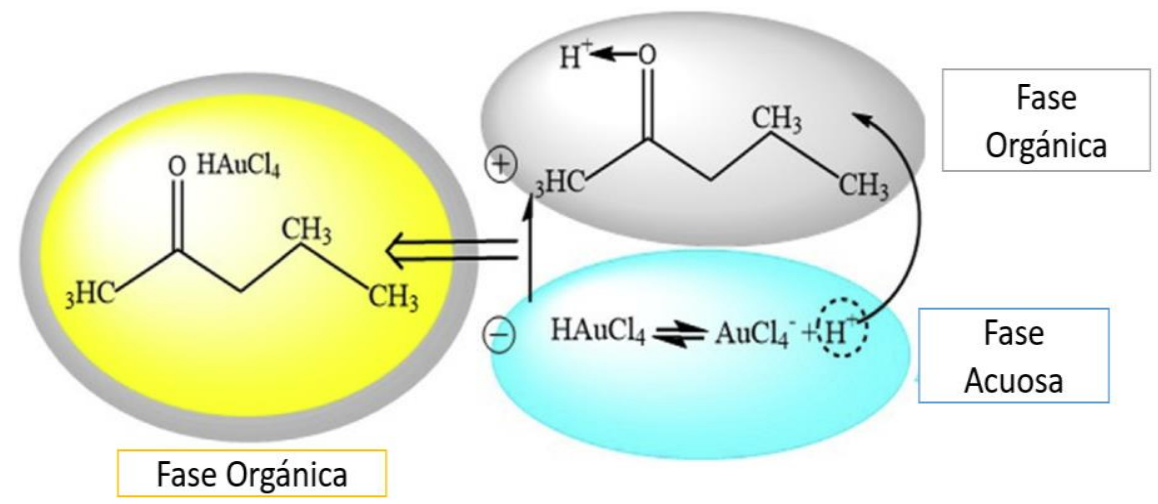

Figura 5. Mecanismo de extracción de oro de una fase acuosa con MIBK. Fuente: [19].

El proceso de extracción utilizando MIBK, ilustrado en la Figura 5, tiene varias ventajas sobre los procesos de extracción convencionales [19]. Durante el proceso de extracción, el átomo de oxígeno en el MIBK proporciona el par de electrones para coordinar con el ion de hidrógeno, y de esta manera, formar un catión $(\mathrm{RH}+)$, mientras que los iones metálicos forman un anión complejo $\left(\mathrm{HAuCl}_{4}^{-}\right)$, que forman el par de iones hidrofóbicos por acción electrostática y luego el complejo de asociación de $\left(\mathrm{RHAuCl}_{4}\right)$. Estos procesos transportan iones Au (III), iones desde la fase acuosa a la fase orgánica, la cual luego es llevada a un proceso de separación por destilación para retirar la fase orgánica del sólido enriquecido con Au.

Finalmente, el material sólido resultante de la destilación es sometido a un proceso de calcinación a $500{ }^{\circ} \mathrm{C}$ por 2 horas [19]. En la Figura 6 se describe este proceso de recuperación de Au.

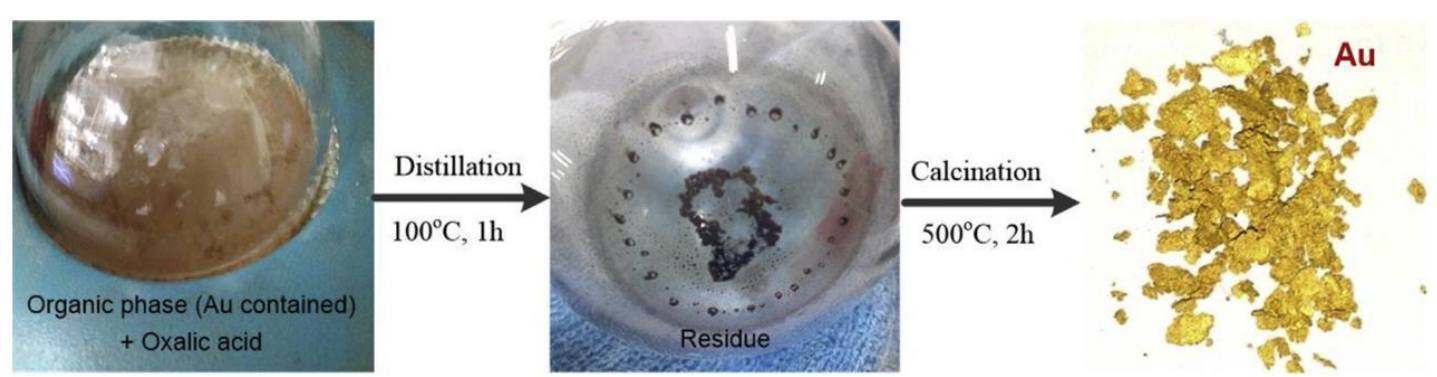

Figura 6. Proceso de refinación del oro (Au) para una recuperación con solventes orgánicos. Fuente: [19].

Las ecuaciones (8), (9), (10) y (11), representan las reacciones químicas involucradas en este tratamiento.

$$
\begin{gathered}
2 \mathrm{Au}_{(\mathrm{s})}+\mathrm{ClO}_{3^{-}(\mathrm{ac})}+6 \mathrm{H}^{+}{ }_{(\mathrm{ac})}+7 \mathrm{Cl}^{-}{ }_{(\mathrm{ac})} \rightarrow 2 \mathrm{AuCl}_{4^{-}(\mathrm{ac})}+3 \mathrm{H}_{2} \mathrm{O}_{(\mathrm{l})} \\
{[R H]_{o}+\left\lceil\mathrm{H}^{+}\right\rceil_{a} \leftrightarrow\left\lceil R H^{+}\right\rceil_{0}} \\
\left.\left\lceil\mathrm{RH}^{+}\right\rceil_{0}+\lceil\mathrm{HAuCl}]_{4}\right\rceil_{a} \leftrightarrow\left\lceil R H A u C l_{4}\right\rceil_{o} \\
2\left\lceil\mathrm{RHAuCl}_{4}\right\rceil_{o}+3 \mathrm{H}_{2} \mathrm{C}_{2} \mathrm{O}_{2} \stackrel{\Delta}{\rightarrow} 2 \mathrm{Au}+2 \mathrm{R}+8 \mathrm{HCl}+6 \mathrm{CO}_{2}
\end{gathered}
$$


Este proceso es continuo, aprovecha subproductos en el mismo y es de lazo cerrado, por lo que las aguas residuales y los gases contaminantes generados son dispuestos correctamente, para evitar daños ambientales y a la salud humana [19].

\section{CASO PRÁCTICO: ANÁlISIS DEL INVENTARIO DEL RECICLAJE DE METALES CONTENIDOS EN TELÉFONOS MÓVILES EN COLOMBIA}

Es escogido este tipo de WEEE, ya que, mundialmente, este es uno de los residuos electrónicos de más rápido crecimiento y de menores porcentajes de recuperación; por ejemplo, entre el 2004 y 2014 fueron vendidos 14 mil millones de unidades de celulares en todo el mundo, de los cuales menos del $3 \%$ fueron recuperados; en países desarrollados como Alemania fue del $5 \% \mathrm{y}$, en China, menos del $1 \%$ [8], [9].

Por otro lado, Colombia cuenta con 25 millones de suscriptores en servicios móviles, ocupando el cuarto puesto de América Latina, por lo que el número de teléfonos importados y vendidos ha ido en continuo crecimiento en el país (Ver Figura 7).

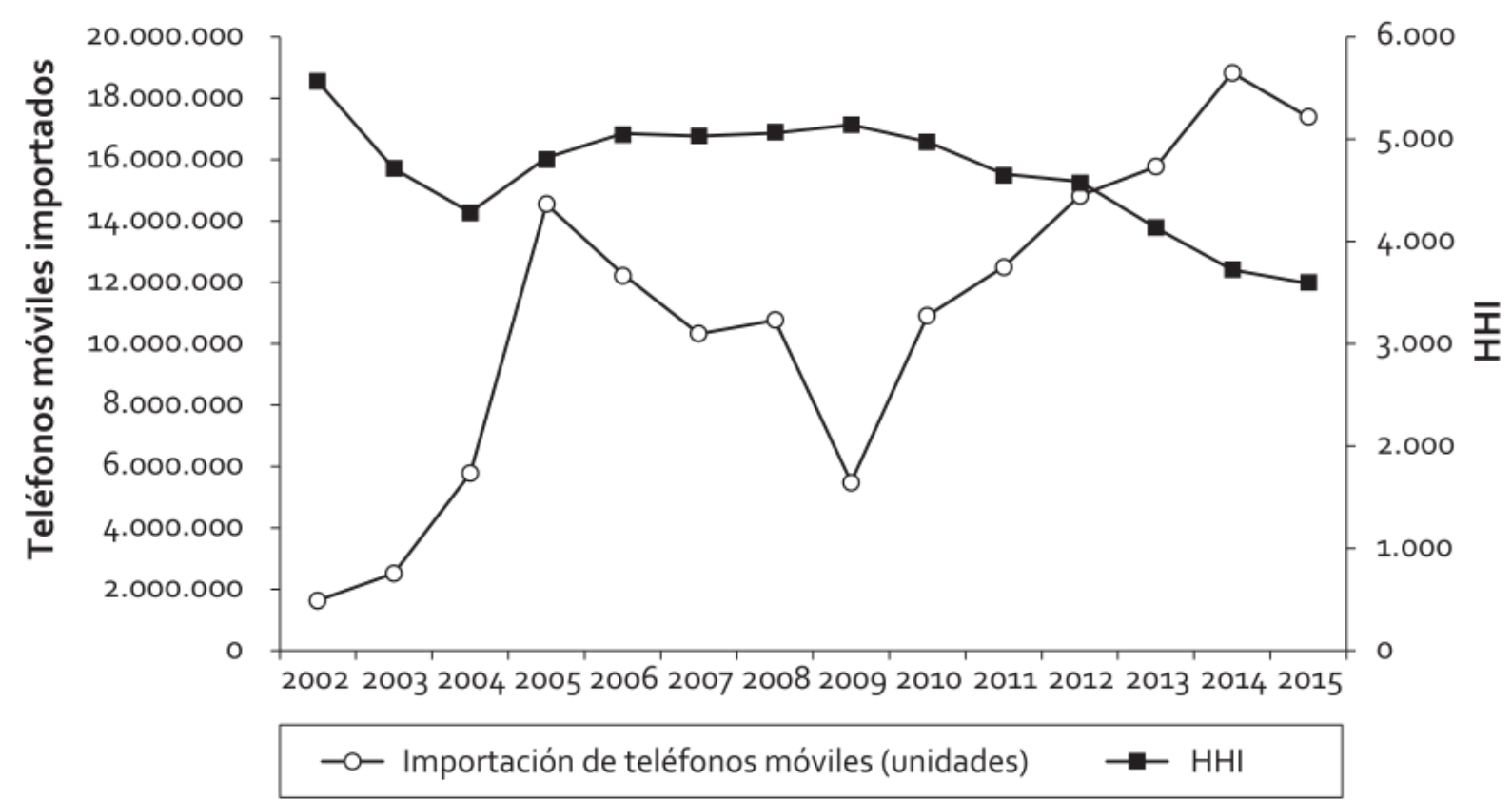

Figura 7. Teléfonos móviles e índice HH del mercado de telefonía móvil en Colombia. Fuente: [15].

Además, según StEP Initiative y el Instituto Tecnológico de Massachusetts (MIT), la vida útil de un teléfono móvil es de 3 años a 5 años [39], lo que aumenta las razones que influyen en el cambio de equipo, entre las cuales también están la obsolescencia y los problemas técnicos [9], [15], sumándose su reincorporación a la cadena como equipo de segunda mano, tal y como sucedió con la iniciativa de "Computadores para Educar", ya que muchos de ellos no se podían actualizar ni reacondicionar [44]. Por lo tanto, se hace más atractivo el proceso de extracción de metales base y preciosos a partir de este tipo de residuos. 


\section{RESULTADOS}

\subsection{Recuperación de Cobre (Cu) y Plata (Ag)}

Los datos ilustrados en la Tabla 4 son los porcentajes y las cantidades de Plata y Cobre presentes en los residuos electrónicos representados en este caso por los dispositivos móviles desechados, y en la Tabla 5 aparecen los kilogramos de Ag y $\mathrm{Cu}$ recuperados por año, de acuerdo con la estequiometria de las reacciones químicas, representadas por (1), (2) y (3).

Además, en ambas tablas se establecen los consumos de las materias primas para la separación y recuperación de estos dos metales a partir de estos WEEE.

Tabla 4. Cantidad de $\mathrm{Cu}$ y Ag en los dispositivos móviles, y el consumo de reactivos por año en la reacción (Rxn) 1 para el caso colombiano. Fuente: elaboración propia.

\begin{tabular}{cccccc}
\hline $\begin{array}{c}\text { Cu en WEEE } \\
(\% \mathrm{w} / \mathrm{w})\end{array}$ & $\begin{array}{c}\mathrm{Cu} \text { en WEEE } \\
(\mathrm{kg})\end{array}$ & $\begin{array}{c}\mathrm{Ag} \text { en WEEE } \\
(\mathrm{ppm})\end{array}$ & $\begin{array}{c}\mathrm{Ag} \text { en WEEE } \\
(\mathrm{kg})\end{array}$ & $\begin{array}{c}\text { Peróxido }\left(\mathrm{H}_{2} \mathrm{O}_{2}\right) \\
\text { consumidos Rxn } \\
1(\mathrm{~kg} / \mathrm{año})\end{array}$ & $\begin{array}{c}\text { Ac Sulfúrico } \\
\left(\mathrm{H}_{2} \mathrm{SO}_{4}\right) \text { consumidos } \\
\text { Rxn 1 (kg/año) }\end{array}$ \\
\hline 13,00 & 24050,00 & 1380,00 & 255,30 & 6437,14 & 18560,93 \\
\hline
\end{tabular}

Tabla 5. Cantidad de $\mathrm{Cu}$ y Ag recuperado de los dispositivos móviles por año, y el consumo de reactivos por año en las reacciones (Rxn) 2 y 3 para el caso colombiano. Fuente: elaboración propia.

\begin{tabular}{ccccc}
\hline $\begin{array}{c}\text { Cobre }(\mathrm{Cu}) \\
\text { recuperado } \\
\text { (kg/año) }\end{array}$ & $\begin{array}{c}\text { Hierro (Fe) } \\
\text { consumidos Rxn } 3 \\
\text { (kg/año) }\end{array}$ & $\begin{array}{c}\text { Plata (Ag) } \\
\text { recuperada como } \\
\text { AgCl (kg/año) }\end{array}$ & $\begin{array}{c}\text { Ag recuperado por } \\
\text { año (kg/año) }\end{array}$ & $\begin{array}{c}\text { NaCl consumidos } \\
\text { Rxn 2 (kg/año) }\end{array}$ \\
\hline 3807,92 & 3523,03 & 56,53 & 42,55 & 23,05 \\
\hline
\end{tabular}

\subsection{Recuperación de Paladio (Pd)}

Los datos ilustrados en la Tabla 6, muestran los kilogramos de Paladio (Pd) presentes en los WEEE analizados y su cantidad recuperada por año, de acuerdo con la estequiometria de las reacciones químicas, ilustradas en (4) y (5). También en esta tabla se pueden observar las cantidades necesarias de reactivos para la separación y recuperación de este metal precioso.

Tabla 6. Cantidades de Paladio $(\mathrm{Pd})$ recuperado de los dispositivos móviles por año, y el consumo de reactivos por año en las reacciones (Rxn) 4 y 5 para el caso colombiano. Fuente: elaboración propia.

\begin{tabular}{|c|c|c|c|c|c|c|}
\hline $\begin{array}{l}\text { Pd en } \\
\text { WEEE } \\
(\mathrm{ppm})\end{array}$ & $\begin{array}{c}\text { Pd en } \\
\text { WEEE (kg) }\end{array}$ & $\begin{array}{l}\text { Paladio }(\mathrm{Pd}) \\
\text { recuperado } \\
\text { por año } \\
(\mathrm{kg} / \mathrm{año})\end{array}$ & $\begin{array}{c}\text { Ac Nítrico } \\
\text { (HNO3) } \\
\text { consumidos } \\
\text { Rxn } 4 \text { (kg/año) }\end{array}$ & $\begin{array}{c}\text { Ac Clorhídrico } \\
\text { (HCl) } \\
\text { consumidos } \\
\text { Rxn } 4 \text { y } 5 \\
\text { (kg/año) }\end{array}$ & $\begin{array}{c}\text { Peróxido (H2O2) } \\
\text { consumidos Rxn } 5 \\
\text { (kg/año) }\end{array}$ & $\begin{array}{c}\mathrm{NaCl} \\
\text { consumidos } \\
\text { Rxn } 4 \text { y } 5 \\
\text { (kg/año) }\end{array}$ \\
\hline 210 & 38,85 & 6,02 & 1,28 & 4,44 & 1,03 & 7,11 \\
\hline
\end{tabular}

\subsection{Recuperación de Oro (Au)}

Finalmente, en la Tabla 7 se ilustran las cantidades Oro (Au) contenidos en los teléfonos móviles desechados en Colombia, los resultados con el Au recuperado a través de un proceso de óxido-reducción, representado por la reacción 6, y las cantidades de químicos necesarios para efectuar dicha reacción química. 
Tabla 7. Cantidades de Oro (Au) recuperado de los dispositivos móviles por año, y el consumo de reactivos por año en la reacción (Rxn) 6 para el caso colombiano. Fuente: elaboración propia.

\begin{tabular}{ccccc}
\hline $\begin{array}{c}\text { Au en WEEE } \\
(\mathrm{ppm})\end{array}$ & $\begin{array}{c}\text { Au en WEEE } \\
(\mathrm{kg})\end{array}$ & $\begin{array}{c}\text { Au recuperado } \\
\text { por año (kg/año) }\end{array}$ & $\begin{array}{c}\text { Tiosulfato de Amonio } \\
\text { consumidos Rxn 6 } \\
(\mathrm{kg} / \mathrm{año})\end{array}$ & $\begin{array}{c}\text { Oxigeno (O2) consumidos } \\
\text { Rxn 6 (kg/año) }\end{array}$ \\
\hline 350 & 64,75 & 10,04 & 16,22 & 0,438 \\
\hline
\end{tabular}

\section{CONCLUSIONES}

Dado el acelerado avance de la tecnología, la generación de residuos de equipos eléctricos y electrónicos va en aumento, lo que a su vez ha provocado que la vida útil de estos dispositivos sea más corta, lo que, al mismo tiempo, se está convirtiendo en un problema mundial por el potencial daño que le pueden ocasionar al ambiente los contaminantes que contienen, como por ejemplo los metales pesados y los volúmenes tan altos que dificultan una correcta disposición final, por lo que se hace imperativo buscar soluciones innovadoras, rentables y sostenibles que generen el mayor valor agregado y que posibiliten una adecuada disposición final de estos residuos.

La recuperación de metales preciosos y de interés para la industria, a partir de WEEE, es una alternativa viable, innovadora y rentable dado que valoriza y le da un segundo uso a un residuo, permitiendo obtener, mediante el reciclaje, materias primas fundamentales para la actividad humana, en este caso, metales de todo tipo, sin la necesidad de explotar las fuentes naturales de estas. El proceso más adecuado para la recuperación de metales a partir de residuos de dispositivos eléctricos y electrónicos es emplear primero uno físico, seguido de uno hidrometalúrgico, pues este proceso es el más amigable y eficiente con el ambiente, con el cual se obtienen los mejores rendimientos en la recuperación de metales. Sin embargo, se debe seguir estudiando e investigando este proceso, porque algunos reactivos utilizados durante la recuperación y/o vertimiento de subproductos aún son considerados tóxicos y dañinos para la salud humana y el ambiente.

Colombia es un gran generador de residuos eléctricos y electrónicos en la región. Sin embargo, la política actual para el manejo posconsumo y disposición final de los WEEE solo tiene en cuenta la recolección y entrega de estos dispositivos al fabricante, sin retribución alguna, lo que desconoce el valor potencial que estos residuos tendrían si son sometidos a algún tratamiento de recuperación de metales preciosos y de interés industrial, por lo que sería una gran oportunidad para el país por la generación de valor agregado en la consolidación de un nuevo tipo de industria rentable y sostenible, y en la disminución de la actividad minera convencional, la cual le hace tanto daño al bienestar de las personas y al medio ambiente.

\section{AGRADECIMIENTOS}

Los autores expresan su agradecimiento al Instituto Tecnológico Metropolitano de Medellín (ITM) y CEA agradece a Sapiencia por su apoyo, a través de la media beca de maestría, dentro del programa Extendiendo Fronteras.

El proyecto no contó con apoyo financiero por parte de ninguna entidad para el desarrollo de esta investigación. 


\section{CONFLICTO DE INTERÉS}

Los autores expresan que no tienen ningún conflicto de interés.

\section{CONTRIBUCIÓN DE AUTORES}

Los autores contribuyeron en la misma medida, ya que al ser un trabajo teórico y de revisión lo plasmado en el presente artículo es el resultado de las discusiones y trabajo mancomunado de los autores.

\section{REFERENCIAS}

[1] M. Ghodrat; M. A. Rhamdhani; G. Brooks; M. Rashidi; B. Samali, "A thermodynamic-based life cycle assessment of precious metal recycling out of waste printed circuit board through secondary copper smelting," Environ. Dev., vol. 24, pp. 36-49, Dec. 2017. https://doi.org/10.1016/j.envdev.2017.07.001

[2] A. Priya; S. Hait, "Toxicity characterization of metals from various waste printed circuit boards," Process Saf. Environ. Prot., vol. 116, pp. 74-81, May. 2018. https://doi.org/10.1016/j.psep.2018.01.018

[3] A. Işildar; E. R. Rene; E. D. van Hullebusch; P. N. L. Lens, "Electronic waste as a secondary source of critical metals: Management and recovery technologies," Resour. Conserv. Recycl., vol 135. pp. 296-312, Agu. 2018. https://doi.org/10.1016/j.resconrec.2017.07.031

[4] M. Kaya, Current WEEE recycling solutions. Elsevier Ltd, 2018.

[5] A. Tuncuk; V. Stazi; A. Akcil; E. Y. Yazici; H. Deveci, "Aqueous metal recovery techniques from e-scrap: Hydrometallurgy in recycling," Miner. Eng., vol. 25, no. 1, pp. 28-37, Jan. 2012. https://doi.org/10.1016/j.mineng.2011.09.019

[6] L. A. Diaz; T. E. Lister, "Economic evaluation of an electrochemical process for the recovery of metals from electronic waste," Waste Manag., vol. 74, pp. 384-392, Apr. 2018.

https://doi.org/10.1016/j.wasman.2017.11.050

[7] I. M. S. K. Ilankoon; Y. Ghorbani; M. N. Chong; G. Herath; T. Moyo; J. Petersen, "E-waste in the international context - A review of trade flows, regulations, hazards, waste management strategies and technologies for value recovery," Waste Manag., vol. 82, pp. 258-275, Dec. 2018. https://doi.org/10.1016/j.wasman.2018.10.018

[8] J. Li; Z. Ge; C. Liang; N. An, "Present status of recycling waste mobile phones in China: a review," Environ. Sci. Pollut. Res., vol. 24, no. 20, pp. 16578-16591, May. 2017. https://doi.org/10.1007/s11356-017-9089-z

[9] N. Gurita; M. Fröhling; J. Bongaerts, "Assessing potentials for mobile/smartphone reuse/remanufacture and recycling in Germany for a closed loop of secondary precious and critical metals," J. Remanufacturing, vol. 8, pp. 1-22, 2018. https://doi.org/10.1007/S13243-018-0042-1

[10] M. Goosey; R. Kellner, "Recycling technologies for the treatment of end of life printed circuit boards (PCBs)," Circuit World, vol. 29, no. 3, pp. 33-37, Sep. 2003. https://doi.org/10.1108/03056120310460801

[11] J. Li; B. N. Lopez; L. Liu; N. Zhao; K. Yu; L. Zheng, "Regional or global WEEE recycling. Where to go?," Waste Manag., vol. 33, no. 4, pp. 923-934, 2013. https://doi.org/10.1016/j.wasman.2012.11.011

[12] A. Akcil; C. Erust; C. Sekhar Gahan; M. Ozgun; M. Sahin; A. Tuncuk, "Precious metal recovery from waste printed circuit boards using cyanide and non-cyanide lixiviants - A review," Waste Manag., vol. 45, pp. 258271, Nov. 2015. https://doi.org/10.1016/j.wasman.2015.01.017

[13] P. Stuhlpfarrer; S. Luidold; H. Antrekowitsch, "Recycling of waste printed circuit boards with simultaneous enrichment of special metals by using alkaline melts : A green and strategically advantageous solution," $J$. Hazard. Mater., vol. 307, pp. 17-25, Apr. 2016. https://doi.org/10.1016/j.jhazmat.2015.12.007

[14] R. Cayumil; R. Khanna; R. Rajarao; P. S. Mukherjee; V. Sahajwalla, "Concentration of precious metals during their recovery from electronic waste," Waste Manag., vol. 57, pp. 121-130, Nov. 2016. https://doi.org/10.1016/j.wasman.2015.12.004

[15] D. Ruiz; S. Bautista, "Factores influyentes en la generación de residuos de teléfonos móviles. Caso Colombia" Gestión y Ambient., vol. 19, no. 2, pp. 228-239, Ago. 2016. URL

[16] F. Fizaine, "The economics of recycling rate: New insights from waste electrical and electronic equipment," Resour. Policy, vol. 67, pp. 101675, Aug. 2020. https://doi.org/10.1016/j.resourpol.2020.101675

[17] Y. Lu; Z. Xu, "Precious metals recovery from waste printed circuit boards : A review for current status and perspective," Resources, Conserv. Recycl., vol. 113, pp. 28-39, Oct. 2016. 
https://doi.org/10.1016/j.resconrec.2016.05.007

[18] C. Hagelüken; C. W. Corti, "Recycling of gold from electronics: Cost-effective use through 'design for recycling," Gold Bull., vol. 43, no. 3, pp. 209-220, Sep. 2010.https://doi.org/10.1007/BF03214988

[19] Y. Lu; Q. Song; Z. Xu, "Integrated technology for recovering Au from waste memory module by chlorination process: Selective leaching, extraction, and distillation," J. Clean. Prod., vol. 161, pp. 30-39, Sep. 2017. https://doi.org/10.1016/j.jclepro.2017.05.033

[20] A. K. Awasthi; J. Li, "An overview of the potential of eco-friendly hybrid strategy for metal recycling from WEEE," Resour. Conserv. Recycl., vol. 126, no. November 2017 pp. 228-239, Nov. 2017. https://doi.org/10.1016/j.resconrec.2017.07.014

[21] H. Oliveros Gómez, "Metodología para recuperar metales preciosos: oro, plata y grupo del platino, presentes en desechos electrónicos,"(Tesis de Maestría), Universidad Nacional de Colombia, Medellín, 2011. URL

[22] A. Gurgul; W. Szczepaniak; M. Zabłocka-Malicka, "Incineration and pyrolysis vs. steam gasification of electronic waste," Sci. Total Environ., vol. 624, pp. 1119-1124, May. 2018. https://doi.org/10.1016/j.scitotenv.2017.12.151

[23] M. Ghodrat; M. A. Rhamdhani; G. Brooks; S. Masood; G. Corder, "Techno economic analysis of electronic waste processing through black copper smelting route,” J. Clean. Prod., vol. 126, pp. 178-190, Jul. 2016, https://doi.org/10.1016/j.jclepro.2016.03.033

[24] G. Chauhan; P. R. Jadhao; K. K. Pant; K. D. P. Nigam, "Novel technologies and conventional processes for recovery of metals from waste electrical and electronic equipment: Challenges \& opportunities - A review," J. Environ. Chem. Eng., vol. 6, no. 1, pp. 1288-1304. Feb. 2018. https://doi.org/10.1016/j.jece.2018.01.032

[25] Ministerio de ambiente y desarrollo sostenible, Decreto 284. Por el cual se adiciona el Decreto 1076 de 2015 , Único Reglamentario del Sector Ambiente y Desarrollo Sostenible, en lo relacionado con la Gestión Integral de los Residuos de Aparatos Eléctricos y Electrónicos - RAEE Y se dictan otras disposiciones, 2015, pp. 110. URL

[26] M. Oguchi; H. Sakanakura; A. Terazono, "Toxic metals in WEEE: characterization and substance flow analysis in waste treatment processes.," Sci. Total Environ., vol. 463-464, pp. 1124-1132, Oct. 2013. https://doi.org/10.1016/j.scitotenv.2012.07.078

[27] M. Bigum; L. Brogaard; T. H. Christensen, "Metal recovery from high-grade WEEE: A life cycle assessment," J. Hazard. Mater., vol. 207-208, pp. 8-14, Mar. 2012. https://doi.org/10.1016/j.jhazmat.2011.10.001

[28] J. Romero Montenegro, "Colombia vs. la basura electrónica, un partido que va empatado," (Trabajo de grado), Universidad del Rosario, 2014. URL

[29] L. Zhang; Z. Xu, "A Review of Current Progress of Recycling Technologies for Metals from Waste Electrical and Electronic Equipment," J. Clean. Prod., vol. 127, pp. 19-36, Jul. 2016. https://doi.org/10.1016/j.jclepro.2016.04.004

[30] H. A. Arroyo; M. C. Fernández, “Tóxicos ambientales y su efecto sobre el neurodesarrollo," Med. (Buenos Aires), vol. 73, no. suppl 1, pp. 93-102, 2013. URL

[31] Z. Sun et al., "Toward Sustainability for Recovery of Critical Metals from Electronic Waste: The Hydrochemistry Processes," ACS Sustain. Chem. Eng., vol. 5, no. 1, pp. 21-40, Sep. 2016. https://doi.org/10.1021/acssuschemeng.6b00841

[32] M. Desmarais; F. Pirade; J. Zhang; E. R. Rene, "Biohydrometallurgical processes for the recovery of precious and base metals from waste electrical and electronic equipments: Current trends and perspectives," Bioresour. Technol. Reports, vol. 11, p. 100526, Sep. 2020. https://doi.org/10.1016/j.biteb.2020.100526

[33] The World Bank Group, "Commodity markets Outlook," International Bank for Reconstruction and Development / World Bank. Washington DC, USA. A World Bank Report 2018 - Oct, Oct 2018. URL

[34] H. S. Park; Y. J. Kim, "A novel process of extracting precious metals from waste printed circuit boards: Utilization of gold concentrate as a fluxing material," J. Hazard. Mater., vol. 365, Mar. 2019, pp. 659-664, 2018. https://doi.org/10.1016/j.jhazmat.2018.11.051

[35] A. Marra; A. Cesaro; V. Belgiorno, "Separation efficiency of valuable and critical metals in WEEE mechanical treatments," J. Clean. Prod., vol. 186, pp. 490-498, Jun. 2018. https://doi.org/10.1016/j.jclepro.2018.03.112

[36] L. Cardona; P. A. Ortiz; A. Restrepo, "Reciclaje Tecnológico al Servicio de la Ciencia," TecnoLógicas, p. 31, Jun. 2010. https://doi.org/10.22430/22565337.317

[37] V. Sahajwalla; V. Gaikwad, "The present and future of e-waste plastics recycling," Curr. Opin. Green Sustain. Chem., vol. 13, pp. 102-107, Oct. 2018. https://doi.org/10.1016/j.cogsc.2018.06.006

[38] J. Hao; Y. Wang; Y. Wu; F. Guo, "Metal recovery from waste printed circuit boards: A review for current status and perspectives," Resour. Conserv. Recycl., vol. 157, pp. 104787, Jun. 2020. https://doi.org/10.1016/j.resconrec.2020.104787

[39] I. Román, "E-Waste en Colombia: El aporte de los operadores móviles en la reducción de la basura electrónica - Estudio de caso,” GSMA. Feb. 2015. URL 
[40] Ministerio de Ambiente y Desarrollo Sostenible, Política nacional para la gestión integral de los residuos de aparatos eléctricos y electrónicos (RAEE). 2017. URL

[41] J. Burlakovs et al., "On the way to 'zero waste' management: Recovery potential of elements, including rare earth elements, from fine fraction of waste," J. Clean. Prod., vol. 186, pp. 81-90, Jun. 2018. https://doi.org/10.1016/j.jclepro.2018.03.102

[42] A. Serpe, "Green chemistry for precious metals recovery from WEEE" in Waste Electrical and Electronic Equipment Recycling: Aqueous Recovery Methods, 1th ed., St. Louis, Missouri: Elsevier B.V., 2018, pp. 271-332. https://doi.org/10.1016/B978-0-08-102057-9.00011-1

[43] B. Rodríguez; L. A González; N. Reyes; L. S. Reyes; A. F. Torres, "Sistema de gestión de residuos de aparatos eléctricos y electrónicos. Enfoque de dinámica de sistemas,” Sist. Telemática, vol. 11, no. 24, pp. 39-53, 2013. URL

[44] ITU, Cuestión 8/2: Estrategias y políticas para la eliminación o reutilización adecuadas de residuos generados por las telecomunicaciones / TIC. 2017. URL

[45] J. Baptiste Bahers; J. Kim, "Regional approach of waste electrical and electronic equipment (WEEE) management in France," Resour. Conserv. Recycl., vol. 129, pp. 45-55, Feb. 2018. https://doi.org/10.1016/j.resconrec.2017.10.016

[46] A. K. Awasthi; J. Li, "An overview of the potential of eco-friendly hybrid strategy for metal recycling from WEEE,” vol. 126, pp. 228-239, Nov. 2017. https://doi.org/10.1016/j.resconrec.2017.07.014

[47] H. Y. Kang; J. M. Schoenung, "Economic analysis of electronic waste recycling: Modeling the cost and revenue of a materials recovery facility in California," Environ. Sci. Technol., vol. 40, no. 5, pp. 1672-1680, Jan. 2006. https://doi.org/10.1021/es0503783

[48] C. A. Kohl; L. P. Gomes, "Physical and chemical characterization and recycling potential of desktop computer waste, without screen," J. Clean. Prod., vol. 184, pp. 1041-1051, May. 2018. https://doi.org/10.1016/j.jclepro.2018.02.221

[49] M. K. Jaunich; J. DeCarolis; R. Handfield; E. Kemahlioglu-Ziya; S. R. Ranjithan; H. Moheb-Alizadeh, "Lifecycle modeling framework for electronic waste recovery and recycling processes," Resour. Conserv. Recycl., vol. 161, pp. 104841, Oct. 2020. https://doi.org/10.1016/j.resconrec.2020.104841

[50] S. Syed, "Recovery of gold from secondary sources-A review," Hydrometallurgy, vol. 115-116, pp. 30-51, Mar. 2012. https://doi.org/10.1016/j.hydromet.2011.12.012

[51] Y. Ding et al., "Recovery of precious metals from electronic waste and spent catalysts: A review," Resour. Conserv. Recycl., vol. 141, no. August 2018, pp. 284-298, 2019. https://doi.org/10.1016/j.resconrec.2018.10.041

[52] M. Sethurajan et al., 'Recent advances on hydrometallurgical recovery of critical and precious elements from end of life electronic wastes - a review", Critical Reviews in Environmental Science and Technology, vol. 49, no. 3, pp 212-275, Jan. 2019. https://doi.org/10.1080/10643389.2018.1540760

[53] M. Wang; Q. Tan; J. F. Chiang; J. Li, "Recovery of rare and precious metals from urban mines-A review," Front. Environ. Sci. Eng., vol. 11, no. 5, pp. 1-17, Jul. 2017. https://doi.org/10.1007/s11783-017-0963-1

[54] H. S. Park; Y. S. Han; J. H. Park, "Massive Recycling of Waste Mobile Phones: Pyrolysis, Physical Treatment, and Pyrometallurgical Processing of Insoluble Residue," ACS Sustain. Chem. Eng., vol. 7, no. 16, pp. 14119- 14125, Jul. 2019. https://doi.org/10.1021/acssuschemeng.9b02725

[55] N. M. Tue; S. Takahashi; A. Subramanian; S. Sakai; S. Tanabe, "Environmental contamination and human exposure to dioxin-related compounds in e-waste recycling sites of developing countries.," Environ. Sci. Process. Impacts, vol. 15, no. 7, pp. 1326-1331, Jun. 2013. https://doi.org/10.1039/c3em00086a

[56] L. Rocchetti; F. VegliòB; Kopacek; F. Beolchini, "Environmental impact assessment of hydrometallurgical processes for metal recovery from WEEE residues using a portable prototype plant," Environ. Sci. Technol., vol. 47, no. 3, pp. 1581-1588, Jan. 2013. https://doi.org/10.1021/es302192t

[57] A. Alzate; M. E. López; C. Serna, "Recovery of gold from waste electrical and electronic equipment (WEEE) using ammonium persulfate," Waste Manag., vol. 57. pp. 113-120, Nov. 2016. https://doi.org/10.1016/j.wasman.2016.01.043

[58] B. Debnath; R. Chowdhury; S. K. Ghosh, "Sustainability of metal recovery from E-waste," Front. Environ. Sci. Eng., vol. 12, no. 2, pp. 1-12, Mar. 2018. https://doi.org/10.1007/s11783-018-1044-9

[59] P. Evangelopoulos; E. Kantarelis; W. Yang, "Waste Electric and Electronic Equipment: Current Legislations, Waste Management, and Recycling of Energy, Materials, and Feedstocks" in Sustainable Resource Recovery and Zero Waste Approaches, 1th ed., St. Louis, Missouri: Elsevier B.V., 2019, pp. $239-266$. https://doi.org/10.1016/B978-0-444-64200-4.00017-7

[60] V. H. Ha; J. C. Lee; T. H. Huynh; J. Jeong; B. D. Pandey, "Optimizing the thiosulfate leaching of gold from printed circuit boards of discarded mobile phone," Hydrometallurgy, vol. 149, pp. 118-126, Oct. 2014. https://doi.org/10.1016/j.hydromet.2014.07.007

[61] Z. Sun; Y. Xiao; H. Agterhui; J. Sietsma; Y. Yang, "Recycling of metals from urban mines - A strategic 
evaluation," J. Clean. Prod., vol. 112, pp. 2977-2987, Jan. 2016. https://doi.org/10.1016/j.jclepro.2015.10.116

[62] P. Quinet; J. Proost, A. Van. Lierde, "Recovery of precious metals from electronic scrap by hydrometallurgical processing routes," Mining, Metallurgy \& Exploration, vol. 22, no. 1, pp. 17-22, Feb. 2005. https://doi.org/10.1007/BF03403191

[63] Y. Zhang; S. Liu; H. Xie; X. Zeng; J. Li, "Current Status on Leaching Precious Metals from Waste Printed Circuit Boards," Procedia Environ. Sci., vol. 16, pp. 560-568, 2012. https://doi.org/10.1016/j.proenv.2012.10.077 